\title{
Test of the Jellium Model: Photoelectron Spectra of Small Copper Clusters
}

\author{
C.-Y. Cha, G.F.Ganteför and W.Eberhardt \\ Institut für Festkörpexforschung, Forschungszentrum Jülich, D-6170 Jülich, Germany
}

\begin{abstract}
Copper cluster anions in the size range of 1-18 atoms are studied by photoelectron spectroscopy. Using photons of $5.0 \mathrm{eV}$ energy the electronic level structure built from the delocalized s-orbitals of the atoms is revealed. An attempt is made to assign the observed bands to the electronic shells predicted by the jellium model. Even though the general behavior resembles the predictions of this model, the spectra are much more complicated reflecting the discrete atomic geometry of these particles.
\end{abstract}

PACS: $36.90 .+\mathrm{d} ; 33.60 . \mathrm{Cv} ; 31.10 .+\mathrm{z}$

\section{Introduction}

The jellium model [1] is successful explaining several properties of metal clusters with highly delocalized valence electrons. Discontinuities in the size dependence of ionization potentials, stabilities, electron affinities and reactivities are found for shell closings predicted by the model. However, the relationship of the electronic structure with these properties is complicated and no direct information about the level structure can be gained from these experimental data.

Photoelectron spectroscopy mirrors the density of states and is a proper tool for testing the electronic structure. The related technique of photoelectron spectroscopy of anions $[2,3]$ is applied here to clusters in order to overcome the specific problems of mass separation and fragmentation.

\section{Experimental setup}

The apparatus is described in detail elsewhere [4]. The cluster anions are generated using a PACIS (Pulsed Arc Cluster Ion Source) and mass separated by a time of flight mass spectrometer. Within the electron spectrometer a selected bunch of anions of defined mass is nearly stopped by a pulsed electric field and then hit by an UV laser pulse. The deceleration enhances the energy resolution in the photoelectron spectra by reducing the Doppler broadening. The kinetic energy of the detached electrons is measured by the time of flight method using a "magnetic bottle" - type spectrometer.

\section{Results and Discussion}

Fig. 1 displays photoelectron spectra of copper cluster anions in the size range from the atom up to $\mathrm{Cu}_{18}^{-}$. The spectra agree with earlier publications [2] besides the differences in energy resolution or photon energy. All clusters exhibit sharp peaks with widths of about $0.3 \mathrm{eV}$. The experimental resolution varies with the kinetic energy of the electrons and the residual velocity distribution of the decelerated anions, but it is small compared to the observed widths except for $\mathrm{Cu}_{1}^{-}$and $\mathrm{Cu}_{3}^{-}$. We cannot exclude contributions from inpurities like hydrates or oxides in the photoelectron spectra. However, the source is run at ultraclean conditions and in the photoelectron spectra there are no indications for contributions from impurities.

Copper cluster show some properties well known from alkali clusters. Especially the even-odd alternation and the shell closings at $8,18,20$ electrons predicted by the jellium model are well reproduced [5]. Thus, for electronic states of clusters, which correspond to the sorbital of the atom, the jellium model should be valid. However, at higher binding energies the atomic d-states give rise to a high density of states, which develops into the d-band of the bulk. For that reason we try to interpret the spectra shown in Fig. 1 within the jellium shell model restricting this analysis to levels above the d-band.

The spectrum of $\mathrm{Cu}_{1}^{-}$can easily be assigned: the s-orbital filled with two electrons (marked s) at $1.3 \mathrm{eV}$ binding energy (BE) and the spin-orbit split d-orbital (marked D) at $2.7 \mathrm{eV} \mathrm{BE}$. To distinguish the atomic $\mathrm{d}$-orbitals and the bulk $\mathrm{d}$ band from the jellium 1d levels we choose capital characters for the former ones. 
In the dimer, the s-orbital splits into a bonding and an antibonding $\mathrm{s}$-orbital with an occupation of $2: 1$ according to the number of available electrons. Within the jellium model, the antibonding $\sigma^{*}$-orbital (marked p) can also be interpreted as the 1p-level due to the p-like shape of the orbital. The broad feature beyond $3.3 \mathrm{eV}$ (marked D) is assigned to the molecular orbitals created from the atomic d-levels.

In the trimer the $1 \mathrm{p}$ orbital (marked $\mathrm{p}$ ) is shifted towards higher binding energies and filled with 2 electrons. Also, the 1s level (marked s) and the d-band (marked D) are shifted into the same direction. The 1s level exhibits a splitting into 3 sublevels, which can be explained as an effect of s-d hybridization [6].

For $\mathrm{Cu}_{4}^{-}$the jellium 1p orbital has to be filled with 3 electrons. Because the tetramer is not spherical, the 1p level will split into 2 or 3 sublevels. Thus, one expects one level corresponding to the one of the trimer shifted towards higher binding energy plus an additional feature at somewhat lower binding energy filled with one electron. Indeed, there are two features with an intensity ratio $2: 1$ at $1.5 \mathrm{eV}$ and $2.3 \mathrm{eV}$ BE. Both are shifted towards lower BE. This may be explained by the overall shift of the energy scale due to the lower electron affinity of the tetramer.

The third feature at $2.7 \mathrm{eV} \mathrm{BE}$ is difficult to explain within the jellium model. It is more likely a shake up satellite. This type of satellite is known from atoms, e.g. $\mathrm{Cu}_{1}$ exhibits a strong shake up peak at $5.1 \mathrm{eV} \mathrm{BE} \mathrm{[6].}$ Thus, we tentatively assign the features marked $\mathrm{p}$ in the spectrum of $\mathrm{Cu}_{4}^{-}$to sublevels of the $1 \mathrm{p}$ jellium orbital and the feature marked $\mathrm{SU}$ to a shake up line of the peak at $2.3 \mathrm{eV} \mathrm{BE}$. The assignment of the features beyond $3.5 \mathrm{eV}$ (marked s, D) seems very uncertain due to the possible s-d hybridization.

For $\mathrm{Cu}_{5}^{-}$the two $1 \mathrm{p}$-levels are easy to identify (marked $\mathrm{p}$ ). The intensity ratio is 1:1 due to the equally distributed 4 p-electrons. The feature at $3.7 \mathrm{eV}$ might be assigned as the $1 s$ level and beyond $4 \mathrm{eV}$ the d-band dominates.

For low symmetry, the 5 th $1 \mathrm{p}$-electron has to be put into the $3 \mathrm{rd} 1 \mathrm{p}$-sublevel, thus three $1 \mathrm{p}$ features with relative intensities $2: 2: 1$ are expected. Indeed, three features with the correct ratios can be identified in the spectrum of $\mathrm{Cu}_{6}^{-}$(marked p). However, there are small additional lines (marked SU). These can be interpreted again as shake up satellites. The intensity ratio and the quite small energy separation in this case also could reflect a multiplet splitting of the states. Beyond $3.8 \mathrm{eV}$ a featureless high density of states is observed due to the $\mathrm{d}$-band (marked D). The 1s orbital is now merged into the D-band.

For larger clusters, the D-band dominates the spectra beyond $4 \mathrm{eV}$. The onset shifts with increasing size towards higher energies and disappears for larger clusters out of the energy window of our experiment.

The shell closing of the 1p jellium levels occurs at 8 electrons. Therefore, neutral alkali cluster consisting of 8 atoms are believed to be of spherical symmetry and the $1 \mathrm{p}$ sublevels are degenerate. In contrast, the spectrum of $\mathrm{Cu}_{7}^{-}$displays two main features (marked p) similar to
$\mathrm{Cu}_{5}^{-}$and $\mathrm{Cu}_{6}^{-}$. According to the foregoing assignment of the peaks of the smaller clusters all features in the spectrum of $\mathrm{Cu}_{7}^{-}$at binding energies smaller than $3.7 \mathrm{eV}$ must belong to the $1 \mathrm{p}$ orbital. Thus, the spectrum is in disagreement with most of the jellium calculations. Recent ab initio calculations [8] support the jellium model, but predict a more complicated splitting into sublevels. For $\mathrm{Na}_{8}$ a splitting of the 1p-orbital into two sublevels is predicted in agreement with the $\mathrm{Cu}_{7}^{-}$spectrum.

The assignment of the peaks of $\mathrm{Cu}_{7}^{-}$is supported by a comparison with the spectrum of $\mathrm{Cu}_{8}^{-}$. The two peaks labelled $p$ simply shift towards higher binding energy and a single line (marked d) at low binding energy appears according to the single electron filled into the $1 \mathrm{~d}$ level. The ab initio calculations of sodium clusters [8] predict a splitting of the $1 \mathrm{p}$ band into three sublevels for $\mathrm{Na}_{n}$ between $n=9-17$. Indeed, for $\mathrm{Cu}_{7}^{-}$the feature at 2.5 $\mathrm{eV}$ binding energy seems to be a double peak similar to the peak at $3 \mathrm{eV} \mathrm{BE}$ in the spectrum of $\mathrm{Cu}_{8}^{-}$. In the size range from $\mathrm{Cu}_{8}^{-}$to $\mathrm{Cu}_{14}^{-}$two or three lines (marked p) can be assigned as sublevels of the 1p band. Beyond $\mathrm{Cu}_{14}^{-}$the $1 \mathrm{~d}$ orbitals dominate the density of states in the observed energy range and part of the $1 p$ band is shifted to BEs beyond the scope of our experiment.

In the jellium model the filling of the $1 \mathrm{~d}$ orbital with its 10 electrons occurs between $\mathrm{Cu}_{8}^{-}$and $\mathrm{Cu}_{17}^{-}$. The features in the spectra (marked d) are assigned with the assumption that up to $\mathrm{Ca}_{15}^{-}$(=81d-electrons) each couple of electrons simply adds one peak to the photoelectron spectrum. Partially the $1 \mathrm{p}$ and $1 \mathrm{~d}$ sublevels overlap and thus the assignment is ambiguous. For $\mathrm{Cu}_{16}^{-}$ the emission intensity is too high to resolve different peaks.

The shell closing of the $1 \mathrm{~d}$ level occurs for $\mathrm{Cu}_{17}^{-}$. The spectrum is dominated by a single feature consisting of two peaks. The comparison with the spectra of $\mathrm{Cu}_{16}^{-}$ and $\mathrm{Cu}_{18}^{-}$suggests an assignment to a highly degenerate $1 \mathrm{~d}$-level. The degeneracy of the cluster orbitals must correspond to a high geometrical symmetry. For $\mathrm{Cu}_{18}^{-}$the $1 \mathrm{~d}$-band splits again into various sublevels and a single weak peak at low binding energy (marked s) reflects the single electron in the $2 \mathrm{~s}$ orbital.

The shell closing at 18 electrons is much more pronounced with respect to the degree of degeneracy of the electronic sublevels compared to the shell closing at 8 electrons. This might reflect the competition between geometrical effects and electronic symmetry. The symmetry of the electronic shell closing at $\mathrm{Cu}_{7}^{-}$is lowered by the limited geometric symmetry of a 7 atom cluster. With a higher number of atoms, a spherical symmetry is much easier to achieve. Thus, the validity of the jellium model increases with increasing cluster size.

\section{Summary}

Features in the photoelectron spectra of small copper clusters at binding energies smaller than $4 \mathrm{eV}$ are tentatively assigned to electronic shells predicted by the jellium model. A certain degree of agreement is only 


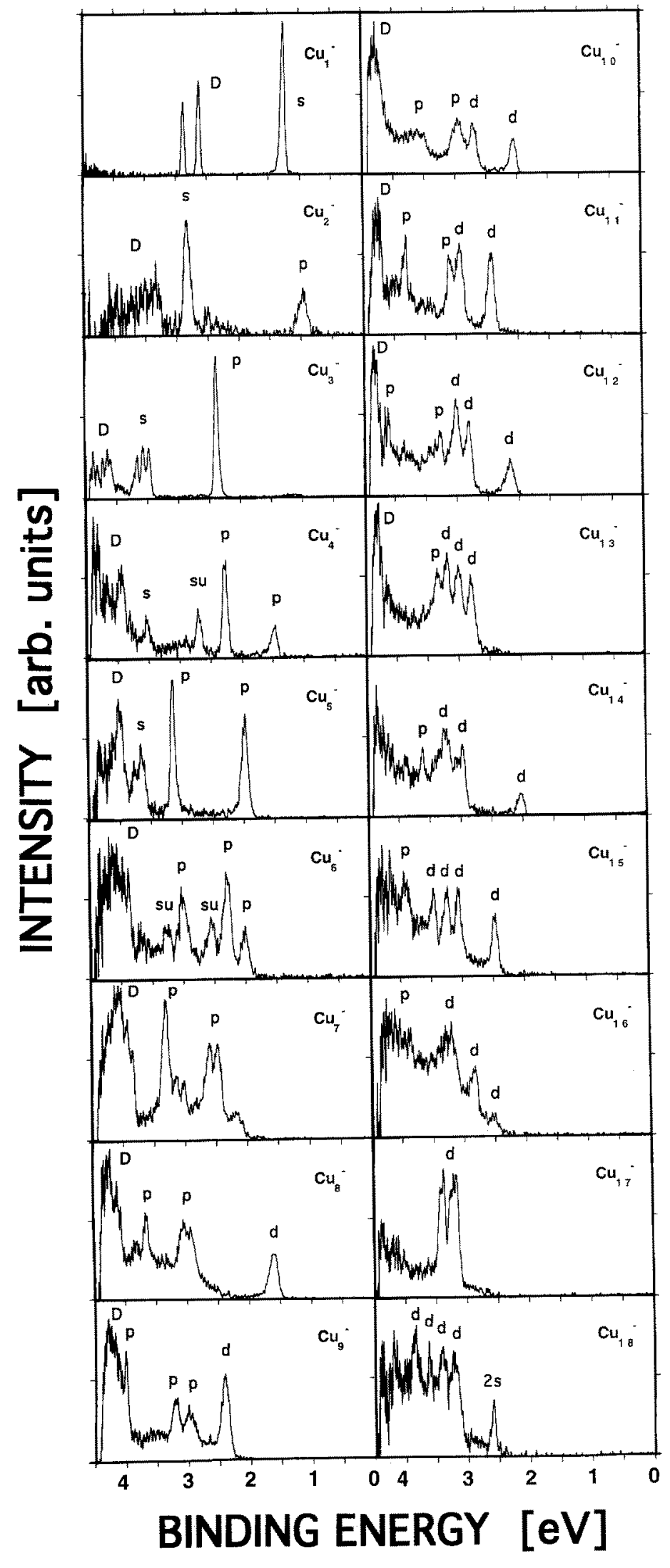

Fig. 1. Photoelectron spectra of $\mathrm{Cu}_{n}^{-}$cluster anions with $n=1-18$. The energy resolution varies depending on the cluster mass and the velocity of the electrons between $100-25 \mathrm{meV}$. The photon energy is $5.0 \mathrm{eV}(\mathrm{KrF}$ excimer laser light). For an explanation of the labels see text. achievable, if shake up satellites, multiplet splitting and a splitting into subshells due to the discrete geometry of the particles are taken into account.

\section{References}

1. W.D. Knight, K. Clemenger, W.A. de Heer, W.A. Saunders, M.Y. Chou, and M.L. Cohen, Phys. Rev. Lett 24, 2141(1984); W.Ekardt and Z.Penzar, Phys. Rev. B 38, 4273 (1988).

2. D.G. Leopold, J.H. Ho, W.C. Lineberger, J. Chem. Phys. 98, $6987(1990)$; K.J. Taylor, C.L. Pettiette-Hall, O. Cheshnovsky, and R.E. Smalley, J. Chem. Phys. 96, 3319 (1992).

3. G.F. Gantefor, K.H. Meiwes-Broer, and H.O. Lutz, Phys. Rev. A 37, 2716 (1988); D.W. Arnold, S.E. Bradford, T.N. Kitsopoulos, and D.M. Neumark, J. Chem. Phys. 95, 8753 (1991).

4. C., Y. Cha, G. Ganteför, and W. Eberhardt, Rev. Sei. Instrum., in press.

5. I. Katakuse, T. Ichihara, Y. Fujita, T. Matsuo, T. Sakurai, and M. Matsuda, Int. J. Mass. Spectrom. Ion Phys. 74, 33 (1986).

6. C.-Y. Cha, G. Gantefor, and W. Eberhardt, to be published.

7. G. Gantefor, Ph.D. thesis, University of Bielefeld, Germany (1988).

8. U. Roethlisberger and W. Andreoni, J. Chem. Phys. 94, 8129 (1991). 